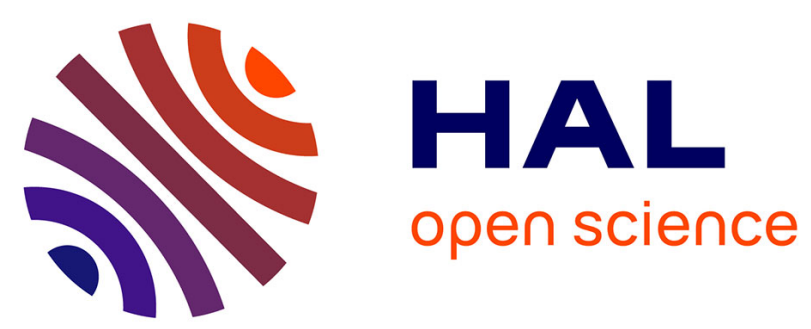

\title{
Clinical and molecular characterisation of hereditary and sporadic metastatic colorectal cancers harbouring microsatellite instability/DNA mismatch repair deficiency
}

R. Cohen, O. Buhard, P. Cervera, Elisabeth Hain, S. Dumont, A. Bardier, J.-B. Bachet, M. Gornet, D. Lopez-Trabada, R. Kaci, et al.

\section{To cite this version:}

R. Cohen, O. Buhard, P. Cervera, Elisabeth Hain, S. Dumont, et al.. Clinical and molecular characterisation of hereditary and sporadic metastatic colorectal cancers harbouring microsatellite instability/DNA mismatch repair deficiency. European Journal of Cancer, 2017, 86, pp.266-274. 10.1016/j.ejca.2017.09.022 . hal-01628456

\section{HAL Id: hal-01628456 \\ https://hal.sorbonne-universite.fr/hal-01628456}

Submitted on 3 Nov 2017

HAL is a multi-disciplinary open access archive for the deposit and dissemination of scientific research documents, whether they are published or not. The documents may come from teaching and research institutions in France or abroad, or from public or private research centers.
L'archive ouverte pluridisciplinaire HAL, est destinée au dépôt et à la diffusion de documents scientifiques de niveau recherche, publiés ou non, émanant des établissements d'enseignement et de recherche français ou étrangers, des laboratoires publics ou privés. 


\section{Original article}

\section{Clinical and molecular characterization of hereditary and sporadic metastatic colorectal cancers harboring microsatellite instability / DNA mismatch repair deficiency}

\section{Authors:}

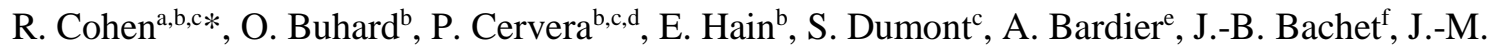

Gornet $^{\mathrm{g}}$, D. Lopez-Trabada ${ }^{\mathrm{a}}$, S. Dumont ${ }^{\mathrm{h}}$, R. Kaci ${ }^{\mathrm{i}}$, P. Bertheau ${ }^{\mathrm{j}}$, F. Renaud ${ }^{\mathrm{k}, \mathrm{lm}}$, F. Bibeau ${ }^{\mathrm{n}}$, Y. Parc ${ }^{\mathrm{b}, \mathrm{c}, \mathrm{o}}$,

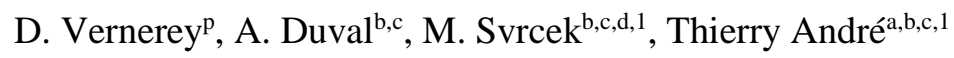

\section{Affiliations:}

${ }^{\text {a }}$ Department of medical oncology, hôpital Saint-Antoine, AP-HP, 184 rue du Faubourg Saint-Antoine, Paris 75012, France

b INSERM, Unité Mixte de Recherche Scientifique 938, Centre de Recherche Saint-Antoine, Equipe Instabilité des Microsatellites et Cancer, Equipe labellisée par la Ligue Nationale contre le Cancer, 184 rue du Faubourg Saint-Antoine, Paris 75012, France

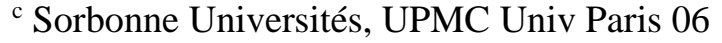

${ }^{\mathrm{d}}$ Department of Pathology, hôpital Saint-Antoine, APHP, 184 rue du Faubourg Saint-Antoine, Paris 75012, France

${ }^{\text {e }}$ Surgical Pathology Department, hôpital Pitié Salpêtrière, Paris, France

${ }^{\mathrm{f}}$ Department of Hepato-Gastroenterology, hôpital Pitié Salpêtrière, Paris, France

g Department of Hepato-Gastroenterology, hôpital Saint-Louis, Paris, France

${ }^{\mathrm{h}}$ Department of medical oncology, Gustave Roussy Cancer Campus, Villejuif, France

${ }^{i}$.Department of pathology, hôpital Lariboisière, Paris, France 
Clinical and molecular characterization of hereditary and sporadic metastatic colorectal cancers harboring microsatellite instability / DNA mismatch repair deficiency

${ }^{j}$ Department of pathology, hôpital Saint-Louis, Paris, France

${ }^{\mathrm{k}}$ Univ. Lille, UMR-S 1172 - JPARC - Jean-Pierre Aubert Research Center, F-59000 Lille, France

${ }^{1}$ INSERM, UMR-S 1172, Team "Mucins, Epithelial Differentiation and Carcinogenesis", F-59000

Lille, France

${ }^{\mathrm{m}}$ CHU Lille, Institut de Pathologie, F-59000 Lille, France

${ }^{\mathrm{n}}$ Department of pathology, Institut du Cancer de Montpellier, Montpellier, France

${ }^{\circ}$ Department of surgery, hôpital Saint-Antoine, Paris, France

${ }^{\mathrm{p}}$ Methodology and quality of life unit in oncology (INSERM UMR 1098), Centre hospitaluniversitaire de Besançon, France

${ }^{1}$ equal contribution

* Corresponding author:

Dr Romain COHEN

Department of medical oncology, hôpital Saint-Antoine, AP-HP, 184 rue du Faubourg Saint-Antoine, Paris 75012, France.

Phone: +33 (0)1 492823 36; Fax: +33 (0)1 492823 44; romain.cohen@aphp.fr 
Clinical and molecular characterization of hereditary and sporadic metastatic colorectal cancers harboring microsatellite instability / DNA mismatch repair deficiency

\section{Abstract:}

Background: Patients treated with chemotherapy for microsatellite-unstable (MSI) and/or mismatch repair-deficient (dMMR) metastatic colorectal cancer (mCRC) exhibit poor prognosis. We aimed to evaluate the relevance of distinguishing sporadic from Lynch syndrome (LS)-like mCRCs.

Patients and methods: MSI/dMMR mCRC patients were retrospectively identified in 6 French hospitals. Tumor samples were screened for MSI, dMMR, RAS/RAF mutations and $M L H 1$ methylation. Sporadic cases were molecularly defined as those displaying MLH1/PMS2 loss of expression with BRAFV600E and/or MLH1 hypermethylation and no MMR germline mutation.

Results: Among 129 MSI/dMMR mCRC patients, 81 (63\%) were LS-like and 48 (31\%) had sporadic tumors; 22\% of MLH1/PMS2-negative mCRCs would have been misclassified using an algorithm based on local medical records (age, Amsterdam II criteria, BRAF and MMR statuses when locally tested), compared to a systematical assessment of MMR, BRAF and $M L H 1$ methylation statuses. In univariate analysis, parameters associated with better overall survival were age $(P<.0001)$, metastatic resection $(P=.001)$ and LS-like $\mathrm{mCRC}(P=.01)$, but not $B R A F \mathrm{~V} 600 \mathrm{E}$. In multivariate analysis, age (hazard ratio $(\mathrm{HR})=3.19, P=.01)$ and metastatic resection $(\mathrm{HR}=4.2, P=.001)$ were associated with overall survival, but not LS. LS-like patients were associated with more frequent liver involvement, metastatic resection and better disease-free survival after metastasectomy $(\mathrm{HR}=.28, P=.01)$. Median progression-free survival of first-line chemotherary was similar between the 2 groups ( 4.2 and 4.2 months; $P=.44)$.

Conclusions: LS-like and sporadic MSI/dMMR mCRCs display distinct natural histories. MMR, BRAF mutation and $M L H 1$ methylation testing should be mandatory to differentiate LS-like and sporadic MSI/dMMR mCRC, to determine in particular whether immune checkpoint inhibitors efficacy differs in these 2 populations. 
Clinical and molecular characterization of hereditary and sporadic metastatic colorectal cancers harboring microsatellite instability / DNA mismatch repair deficiency

\section{Keywords:}

microsatellite instability; mismatch repair; Lynch syndrome; BRAF mutation; immunotherapy; colorectal cancer.

\section{Highlights:}

- Metastatic colorectal cancers with sporadic or inherited mismatch repair deficiency display distinct natural histories.

- A molecular algorithm combining MMR protein expression, BRAF mutation and $M L H 1$ methylation testing is mandatory to properly determine the mechanism underlying mismatch repair deficiency.

- A better characterization of MSI/dMMR CRCs is needed to determine whether the effectiveness of therapeutic agents (chemotherapy, targeted therapies, immune checkpoint inhibitors) differs depending on the origin of the MMR deficiency.

\section{Fundings:}

Fondation ARCAD

\section{Acknowledgement:}

The authors thank Delphine Lees for editorial assistance. 


\section{INTRODUCTION}

Microsatellite instability (MSI) is caused by the deficiency of the mismatch repair (MMR) system, resulting from a germline mutation in MMR genes (MLH1, PMS2, MSH2, MSH6) predisposing to Lynch syndrome (LS), or an epigenetic inactivation of $M L H 1$. Sporadic MSI/MMR-deficient (dMMR) colorectal cancer (CRC), unlike LS-related CRC, is associated with the BRAFV600E mutation, through its association with CIMP (CpG island methylator phenotype) [1]. MMR genes germline mutation cannot always be detected in LS and the definition of sporadic MSI/dMMR CRCs and CRCs presumed to be LS-related (i.e. Lynch-like) has evolved over time, from clinical criteria to a tailored approach with $B R A F$ and $M L H 1$ methylation testing for CRCs showing a loss of MLH1 expression [2-5].

Stage II MSI/dMMR CRC has a favorable prognosis. 5-fluorouracile-based adjuvant chemotherapy (5fluorouracile alone, with leucovorin or levamisol) is ineffective on stage II MSI/dMMR CRCs [6]. However, the addition of oxaliplatine to fluoropyrimidines seems associated with a survival benefit for patients with high-risk stage II or stage III MSI/dMMR CRCs [7,8]. Sensitivity to fluoropyrimidine may differ between sporadic and hereditary stage III MSI/dMMR CRCs $[8,9]$. In metastatic setting, the poor prevalence of MSI/dMMR (3-5\%) hampers the evaluation of its prognostic value, and results from early studies were inconsistent [10-14]. The pooled analysis of four phase III studies in first-line treatment of mCRC demonstrated that dMMR is associated with reduced progression-free survival (PFS) and overall survival (OS), with an insignificant negative effect of BRAFV600E among MSI mCRCs [15]. Thus, the impact of BRAFV600E on MSI CRCs remains controversial.

While immune checkpoint inhibitors (ICK) have demonstrated promising efficacy for MSI tumors, unresolved issues persist concerning molecular and clinical heterogeneity among MSI/dMMR mCRC patients $[16,17]$. In our multicentre retrospective study, we aimed to evaluate a molecular definition of sporadic and inherited MMR deficiency of MSI/dMMR mCRC patients, with a particular interest in its clinical relevance before the era of immunotherapy. 


\section{MATERIAL AND METHODS}

\section{Study population}

This retrospective study was conducted in 6 French hospitals. All patients with locally determined MSI and/or dMMR mCRC were eligible. Tumor specimen collection was mandatory. Exclusion criteria were: no tumor specimen available, MMR-proficient (pMMR) and microsatellite stable (MSS) tumors. Ethical approval was provided by an institutional review board (Comité de Protection des Personnes Ile-de-France IV, N IRB 00003835) on November 27, 2014.

\section{Clinical data}

All medical and pathological records were analyzed for demographic and clinical characteristics, treatment received and survival. CRCs arising in the splenic flexure, descending colon, sigmoid colon or rectum were classified as left-sided; those originating in cecum, ascending colon, hepatic flexure or transverse colon were classified as right-sided.

\section{$\underline{\text { Tumor tissue analysis }}$}

All tumor specimen were tested for MMR and microsatellite statuses. MLH1 promoter methylation status was assessed for MLH1/PMS2-negative tumors. KRAS, NRAS and BRAF mutational statuses were evaluated [supplementary methods].

\section{Determination of sporadic and Lynch syndrome-associated dMMR/MSI tumors}

When the germline MMR gene mutation analysis was not available, patients were considered to have suspected LS if their tumor exhibited a loss of expression of MSH2/MSH6, MSH6 alone or PMS2 alone, or a loss of MLH1/PMS2 expression without BRAF mutation and no MLH1 hypermethylation. MLH1/PMS2-negative tumors harboring BRAF mutation or $M L H 1$ hypermethylation were considered sporadic [18]. Similarly, sporadic or hereditary origin of MSI tumors with no loss of MMR protein expression was determined according to $B R A F$ mutation and $M L H 1$ methylation [figure S1]. 
As comparator, an unsystematic molecular definition that only takes into account data provided by local pathological and medical records was also used (i.e. MMR testing and $B R A F$ status when available; if not, age inferior to 60 years or Amsterdam II criteria) $[8,9]$.

\section{$\underline{\text { Statistical analysis }}$}

Median value (interquartile range) and frequency (percentage) were provided for the description of continuous and categorical variables, respectively. Medians and proportions were compared according to hereditary or sporadic origin using Student's t test and chi-square test (or Fisher's exact test, if appropriate), respectively.

For patients with initially stage I-III tumors, disease-free survival (DFS) was calculated from primary tumor surgery to relapse (second CRC, loco-regional or metastatic relapse) or death. Overall survival (OS) was defined as the time between the diagnostic of metastatic disease to the date of death as a result of any cause. Patients treated with ICK in clinical trials were excluded from OS analysis. For patients with resected metastatic disease, DFS was calculated from the date of metastasis resection to the date of relapse or death. Progression-free survival (PFS) was calculated from the date of first-line chemotherapy initiation for metastatic disease to the date of tumor progression or death from any cause; patients with metastasectomy during chemotherapy course were excluded from PFS analysis. Survival curves were estimated using the Kaplan-Meier method and compared with a Log-rank test. Follow-up was calculated by reverse Kaplan-Meier estimation. .

Cox proportional hazard models were performed to estimate hazard ratio (HR) and 95\% confidence interval $(95 \% \mathrm{CI})$ for factors associated with OS. The association of baseline parameters with OS was first assessed using univariate Cox analyses, parameters with $P$ values $<.05$ were then entered into a final multivariate Cox regression model after considering collinearity among variables with a correlation matrix.

All analyses were performed using SAS version 9.4 (SAS Institute, Cary NC) and R software version 2.15.2 (R Development Core Team, Vienna, Austria; http://www.r-project. org). P values of less than .05 were considered statistically significant, and all tests were two-sided. 


\section{RESULTS}

\section{$\underline{\text { Population }}$}

Among 160 patients identified with dMMR and/or MSI mCRC, 31 were excluded: no tumor sample available $(N=15)$, pMMR and MSS tumors according to centrally analysis $(N=16)$. The 129 remaining patients had been diagnosed with mCRC between January 1998 and January 2016.

\section{Distinction between sporadic and Lynch-like tumors}

Results of MMR immunohistochemistry, BRAF mutation and $M L H 1$ methylation analysis are presented in table 1. Forty-eight patients (37\%) had sporadic dMMR/MSI mCRC and 81 (63\%) were presumed to be Lynch-like, according to the molecular approach combining MMR, $M L H 1$ methylation and $B R A F$ mutation testing. Of note, 18 out of all 48 sporadic MSI/dMMR mCRCs (38\%) were BRAF wild-type but $M L H 1$ hypermethylated.

A MMR gene germline mutation was known for 34 patients (26\%). No BRAF mutation or MLHI hypermethylation were detected in the tumors of these 34 Lynch syndrome-proven patients.

$22 \%$ of MLH1 and/or PMS2 negative mCRCs with no known MMR germline mutation (14/64) would have been misclassified using an unsystematic molecular approach. Taking into account local pathological records only, in the absence of our systematic analysis of BRAF mutation and $M L H I$ methylation, 9 Lynch-like tumors would have been considered as sporadic and 5 sporadic MSI/dMMR mCRCs as Lynch-like [table S1].

Clinical and molecular characteristics

Demographic and clinical characteristics are summarized in table 2. Median age at diagnosis of mCRC was 57 years. Among initially localized MSI/dMMR mCRCs $(N=69), 33 \%$ were stage II and $67 \%$ stage III. Main first metastatic sites were: peritoneum (46\%), liver (38\%), distant lymph nodes (33\%) and lung (2\%). 
Patients with sporadic MSI/dMMR mCRC were associated with female $(P<0.001)$, older age at diagnosis $(P<0.0001)$, right-sided primary tumor $(P=0.02)$ and less liver involvement $(P=0.02)$, with a trend for synchronous metastatic disease ( $56 \%$ versus $41 \%, P=0.10)$. A sex ratio of 3.1 (male/female) was observed for patients with Lynch-like mCRC.

Thirty-three per cent, $3 \%$ and $23 \%$ of all MSI/dMMR mCRCs harbored a mutation of KRAS, NRAS and $B R A F$, respectively. Forty-five tumors (35\%) were all wild-type.

\section{Resection of metastatic disease}

Resection of metastatic disease was reported in 54\% of Lynch-like mCRCs $(N=44 / 81)$ and $21 \%$ of sporadic tumors $(N=10 / 48) ; P<0.001)$. Resected metastases were located in peritoneum only $(N=$ $20)$, liver only $(N=19)$, distant lymph nodes only $(N=6)$, multi-sites $(N=5)$ or others $(N=4)$. Among those who relapsed $(N=48)$, metastatic resection was reiterated at least once for 16 patients (10 Lynch-like, 2 sporadic), 10 of which did not relapse during follow-up. At the data cut-off date, 16 patients (Lynch-like, $N=15$; sporadic, $N=1$ ) were in complete remission.

\section{$\underline{\text { Survival }}$}

\section{Overall survival}

Among 129 MSI/dMMR mCRC patients, 45 were treated with ICK in KEYNOTE-164 and CHECKMATE-142 trials and therefore excluded from OS analysis. Median OS was 39.1 months in the overall population $(N=84)$. Median follow-up was 27 months. Median OS was superior for Lynch-like patients compared to sporadic cases (43.9 months versus 18.5 months; $\mathrm{HR}=0.39,95 \% \mathrm{CI}$ [0.18-0.89], $P=0.008)$ [figure 1].

Results from univariate and multivariate analysis are presented in table 3. In univariate analysis, hereditary origin of MMR deficiency $(\mathrm{HR}=0.39,95 \% \mathrm{CI}[0.19-0.81], P=0.01)$, younger age at diagnosis of metastasis $(P<0.0001)$ and resection of metastatic disease $(P=0.0001)$ were associated with better OS. BRAF mutation was not statistically associated with OS. Multivariate analysis showed that younger age and metastasis resection were associated with OS (HR $=0.314,95 \%$ CI $[0.129$ - 
0.766], $P=0.01$ and $\mathrm{HR}=0.238,95 \% \mathrm{CI}$ [0.100 to 0.564 ], $P=0.001$ ), but not the origin of MMR deficiency $(\mathrm{HR}=1.27,95 \% \mathrm{CI}[0.50-3.20], P=0.62)$. Collinearity was observed between LS, age and metastasis resection (table S2).

Among patients with sporadic MSI/dMMR mCRC, no difference in OS was observed according to $B R A F$ status $(P=0.91)$.

\section{Disease-free survival}

For patients with initially localized mCRC $(N=69)$, median DFS was 11.63 months in the overall population, 9.8 months for sporadic cases and 12.1 months for Lynch-like tumors $(\mathrm{HR}=0.53,95 \% \mathrm{CI}$ [0.74-2.1], $P=.04)$.

For patients with metastatic disease resection $(N=54)$, median DFS after metastasectomy was 9.5 months and was significantly inferior for sporadic than Lynch-like patients (6.7 versus 10.5 months, $\mathrm{HR}=0.28,95 \% \mathrm{CI}[0.1-0.73], P=0.01)$ [figure 1].

\section{$\underline{\text { First-line progression-free survival }}$}

Sixty-three patients received first-line chemotherapy for unresectable metastatic disease. Median PFS was 4.2 months in the overall population, as well as in sporadic and Lynch-like groups [figure 1].

Main chemotherapeutic regimens were: FOLFIRI $(N=26 ; 41 \%), \operatorname{FOLFOX}(N=26 ; 46 \%)$, and FOLFIRINOX $(N=3 ; 5 \%)$. Anti-angiogenic agents and anti-EGFR (epidermal growth factor receptor) were associated with chemotherapy for 24 (38\%) and 12 (19\%) patients, respectively. 


\section{DISCUSSION}

We report here a large cohort dedicated to patients with MSI/dMMR mCRC. Although retrospective, this multicenter study exhibits robustness thanks to the mandatory tumor specimen collection. This biological collection paired to a clinical database allowed a systematic analysis of microsatellite, MMR, BRAF and MLH1 methylation statuses[16][15][16][16].

We show that the determination of MMR deficiency origin using a molecular approach is technically feasible in metastatic setting and efficient, despite poor pre-analysis conditions (i.e. retrospectively collected FFPE samples). Indeed, 22\% of patients with MLH1/PMS2-negative mCRC would have been misclassified using widely-used algorithms combining MMR immunohistochemistry, BRAF mutation, age and Amsterdam II criteria [8,9]. Notably, 38\% of sporadic MSI/dMMR mCRCs were $B R A F$ wild-type but $M L H 1$ hypermethylated. Twenty-five per cent of patients with sporadic MSI/dMMR mCRC were aged 60 or less, and 25\% of Lynch-like patients were older than 55 years, indicating that age is ineffective at distinguishing inherited and sporadic MSI/dMMR mCRCs. Thus, when the germline MMR gene mutation analysis is not available, it is necessary to test MMR protein expression using immunohistochemistry and, for MLH1/PMS2-negative tumors, both BRAF mutation and MLH1 methylation, in order to correctly identify sporadic or Lynch-like MSI/dMMR mCRCs $[18,19]$.

In our cohort, $62 \%$ of MSI/dMMR mCRCs were presumed to be related to Lynch syndrome. This result might be explained by the fact that many participants of this cohort are expert centers for familial colorectal cancers. However, the true proportion of sporadic and inherited tumors among MSI/dMMR mCRCs is unknown. In our study, patients with sporadic MSI/dMMR mCRC were associated with female, older age, right-sided primary tumor and less liver involvement. These results are concordant with previously published works $[12,20]$. Surprisingly, Lynch-like mCRC patients had a sex ratio superior to 3 in favor of male. This trend was observed in a pooled analysis of randomized trials in first-line treatment among $B R A F$ wild-type dMMR mCRC patients (66 males, 33 females) [15]. Further research will have to explore the biological determinants of this finding. 
The inherited origin of MMR deficiency, younger age at diagnosis of mCRC and resection of metastasis were associated with better OS in univariate analysis. Our study being insufficiently powered to compute age as a continuous variable in multivariate analysis, a cut-off of 70 years was used [21]. Younger age and metastatic surgery, but not LS, were associated with OS in multivariate analysis. However collinearity among these variables was observed.

Of note, BRAFV600E was not associated with OS. Moreover, no prognostic difference was observed between $B R A F$-mutated and -wild type sporadic MSI/dMMR mCRC. This enlightens the analysis of Venderbosch and colleagues where the negative prognostic effect of BRAFV600E on OS did not reach statistical significance within MSI/dMMR mCRCs (HR $=1.51,95 \%$ CI 0.93-2.46) [15]. On the contrary, Goldstein et al. reported a statistically significant negative impact of BRAFV600E on OS in a retrospective cohort of $55 \mathrm{MSI} / \mathrm{dMMR}$ mCRCs [22]. In localized setting, BRAFV600E is not associated with prognosis (OS) among stage II and III MSI/dMMR CRCs [21,23]. Since CMS 1 (consensus molecular subtype 1) has high BRAF-mutated and MSI tumors and is characterized by favorable recurrence-free survival but poor survival after relapse, it appears necessary to investigate the clinical and molecular heterogeneity of CMS1 CRCs, with a particular interest in CIMP status, which overlaps with $B R A F$ mutation and MSI phenotype $[24,25]$.

Patients with Lynch-like mCRC underwent metastasis surgery more frequently and exhibited a significantly better median DFS than sporadic MSI/dMMR mCRC patients (HR $=0.28,95 \%$ CI $[0.1$ 0.73], $P=0.01$ ). This might be explained by the fact that Lynch-like mCRCs are associated with younger age and higher frequency of liver metastasis. Moreover, MMR genes mutation carriers are followed with colonoscopy every 1 to 2 years, allowing the early detection of CRC, whereas patients with sporadic MSI/dMMR mCRCs are associated with older age and therefore frequently out of screening programs $[19,26]$.

Median PFS for patients receiving first-line chemotherapy for unresectable mCRC did not differ between sporadic and Lynch-like groups (4.2 months in both groups). This is consistent with the analysis of Venderbosch and colleagues (median PFS of 6.1 and 6.3 months for BRAF-mutated and $B R A F$-wild type MSI mCRC patients, respectively) [15]. The poor median PFS in first-line setting 
Clinical and molecular characterization of hereditary and sporadic metastatic colorectal cancers harboring microsatellite instability / DNA mismatch repair deficiency

with comparison to the analysis of Venderbosch might be partly explained by the retrospective nature of our study. Moreover, since it was retrospectively not possible to properly differentiate patients with resectable or unresectable metastatic disease before the initiation of first-line chemotherapy, we excluded patients with metastasectomy from this PFS analysis.

Nivolumab monotherapy for MSI/dMMR mCRC $(N=74)$ showed promising efficacy in patients with or without clinical history of LS, determined by investigators based on past medical history collected from clinical records [17]. Similarly, favorable outcomes have been reported among BRAFV600Emutated MSI/dMMR mCRCs treated with ICK $[16,17,27]$. However, these results should be taken with caution as the criteria for the distinction between sporadic and LS-related MMR deficiency were unclear. Given the clinical and survival differences observed in our cohort between sporadic and LSrelated MSI/dMMR mCRC patients, the mechanism of the MMR deficiency should be systematically determined in future studies evaluating therapies in this population. An efficient and consensual definition, as we propose here, will be necessary to properly evaluate the efficacy of ICK for patients with MSI/dMMR mCRC with regards on the mechanism underlying MMR deficiency. 


\section{CONCLUSION}

As immunotherapy is on the rise for MSI/dMMR tumors, there is an urge for studies that clinically and molecularly characterize patients with MSI/dMMR mCRCs before the era of ICK. We show that MMR protein expression, BRAF mutation and $M L H 1$ promoter methylation testing are needed to properly distinguish sporadic and inherited MSI/dMMR mCRCs. MSI/dMMR mCRC is a clinically heterogeneous disease associated with poor chemosensitivity. With a distinct pattern of metastatic spread characterized by a higher frequency of liver involvement, patients with Lynch-like mCRC are associated with younger age, more frequent metastatic surgery and favorable prognosis compared to those with sporadic MSI/dMMR mCRC. BRAF mutation, $M L H 1$ methylation and MMR testing will be essential to evaluate whether the efficacy of anticancer treatments differs between sporadic and LSrelated MSI/dMMR mCRC patients. 
Table 1: Molecular distinction between sporadic and Lynch-like tumors

\begin{tabular}{lrrr}
\hline & Overall population & Sporadic group & Lynch-like group \\
& $N(\%)$ & $N$ & $N$ \\
\hline Number of patients & $129(100)$ & 48 & 27 \\
MLH1/PMS2 loss of expression & $71(55)$ & - & 12 \\
$M L H 1$ constitutive mutation & & 27 & - \\
$B R A F^{\mathrm{MT}}$ & & 17 & 15 \\
$B R A F^{\mathrm{WT}}$, hypermethylated & & - & 39 \\
$B R A F^{\mathrm{WT}}$ not hypermethylated & $39(30)$ & - & 9 \\
MSH2/MSH6 loss of expression & $9(7)$ & $3 *$ & 2 \\
Loss of expression of MSH6 alone & $5(4)$ & $1 * *$ & $4 * * *$ \\
Loss of expression of PMS2 alone & $5(4)$ & & \\
pMMR MSI tumors & & & \\
\hline$B R A F^{\mathrm{MT}}: B R A F \mathrm{~V} 600 \mathrm{E}$ & & & \\
\end{tabular}

$B R A F^{\mathrm{MT}}: B R A F \mathrm{~V} 600 \mathrm{E}$ mutated; $B R A F^{\mathrm{WT}}: B R A F$ wild-type;

*: PMS2-negative $B R A F^{\mathrm{MT}} /$ hypermethylated tumors

**: 1 pMMR MSI $B R A F^{\mathrm{MT}}$ tumor

***: 2 pMMR MSI tumors with known MMR gene constitutive mutation, $2 \mathrm{BRAF}^{\mathrm{WT}}$ tumors without hypermethylation 
Table 2: Clinical and molecular characteristics

\begin{tabular}{lllll}
\hline & Overall & Sporadic & Lynch-like & \\
& population & group & group & \\
& $N(\%)$ & $N(\%)$ & $N(\%)$ & $P$ \\
& $129(100)$ & $48(100)$ & $81(100)$ & \\
\hline Number of patients & $1.4(75 / 54)$ & $0.4(14 / 34)$ & $3.1(61 / 20)$ & $<0.001^{*}$
\end{tabular}

Age at diagnosis of metastatic CRC

median, Q1-Q3 (years) $\quad 57,42-68 \quad 71,61-76 \quad 47,36-56 \quad<0.0001 *$

Stage at diagnostic

stage II

stage III

stage IV

Primary tumor plurifocality

Primary tumor location

right

left

left and right**

Primary tumor resection

First metastatic sites

$\geq 2$ organs involved

peritoneum

liver

distant lymph nodes

others

RAS/RAF mutational status***
$46(36)$

$5(4)$

(36)

$23(18)$

$46(36)$

$60(47)$

14 (11)

$86(67)$

$35(27)$

$8(6)$

125 (97)

37 (29)

$56(46)$

49 (38)

$43(33)$

$21(16)$
$6(13)$

$15(31)$

27 (56)

$3(6)$

$39(81)$

$7(15)$

$2(4)$

47 (98)
$78(96) \quad 0.99$

$17(21)$

$31(38)$

$33(41) \quad 0.10$

$11(14) \quad 0.25$

47 (58)

$0.02 *$

$28(35)$

$6(7)$
KRAS mutation

NRAS mutation
7 (15)

$0(0)$
$11(23)$

$22(46)$

$12(25)$

$20(42)$

$5(10)$
$26(32)$

$34(42)$

37 (46)

23 (28)

$16(20)$
0.32

0.72

$0.02 *$

0.13

0.22 
Clinical and molecular characterization of hereditary and sporadic metastatic colorectal cancers harboring microsatellite instability / DNA mismatch repair deficiency

$\begin{array}{lcccc}\text { BRAF mutation } & 30(23) & 30(63) & 0 & <0.001 * \\ \text { all wild-type } & 45(35) & 11(23) & 34(42) & 0.02 * \\ \text { not evaluable } & 5(4) & 1(2) & 4(5) & \end{array}$

*: statistically significant

**: patients with both right-sided and left-sided tumors at diagnostic were classified as "left and right" ***: 2 tumors exhibited 2 distinct mutations (both KRAS and BRAF mutations for 1 tumor; both KRAS and NRAS for the other one) 
Table 3: Univariate and multivariate analysis for overall survival

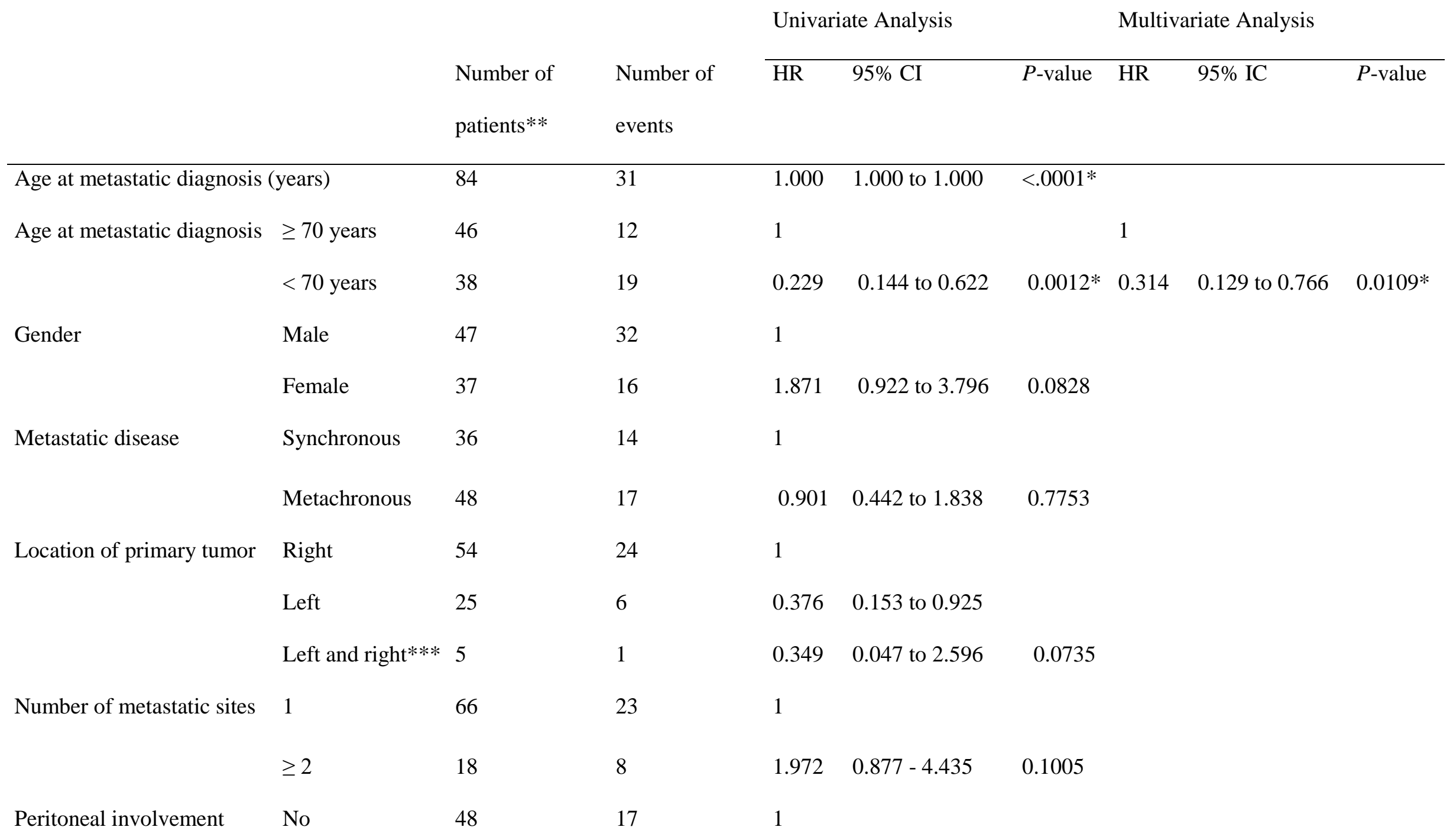




\begin{tabular}{|c|c|c|c|c|c|c|c|c|c|}
\hline & Yes & 36 & 14 & 1.163 & 0.573 to 2.361 & 0.6761 & & & \\
\hline \multirow[t]{2}{*}{ Resection of metastasis } & No & 47 & 22 & 1 & & & 1 & & \\
\hline & Yes & 37 & 9 & 0.208 & 0.094 to 0.460 & $0.0001 *$ & 0.238 & 0.100 to 0.564 & $0.0011^{*}$ \\
\hline \multirow[t]{2}{*}{ BRAFV600E mutation } & wild-type & 64 & 23 & 1 & & & & & \\
\hline & mutated & 20 & 8 & 1.987 & 0.881 to 4.481 & 0.0978 & & & \\
\hline \multirow[t]{2}{*}{ Origin of MMR deficiency } & Sporadic & 34 & 15 & 1 & & & 1 & & \\
\hline & Lynch-like & 50 & 16 & 0.394 & 0.193 to 0.806 & $0.0107 *$ & 1.265 & 0.501 to 3.191 & 0.6185 \\
\hline
\end{tabular}



instability / DNA mismatch repair deficiency

Figure 1: Kaplan-Meier curves of overall population and according to MMR deficiency origin for overall survival (1A), disease-free survival after primary tumor resection (1B) and resection of metastatic disease (1C) and first-line progression-free survival (1D)

*LS-related: Lynch syndrome-related ; Black line: overall population; blue line: sporadic group; red line: Lynch-syndrome-related group

$1 \mathbf{A}$

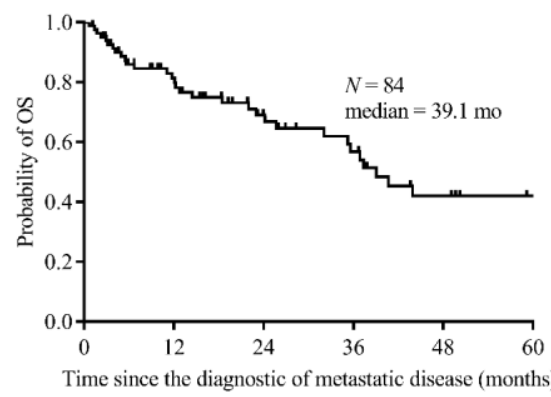

$1 \mathrm{~B}$

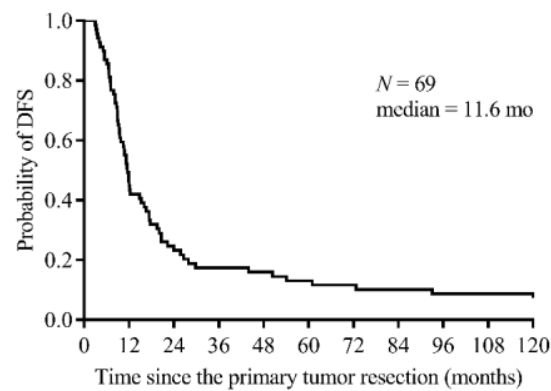

$1 \mathrm{C}$

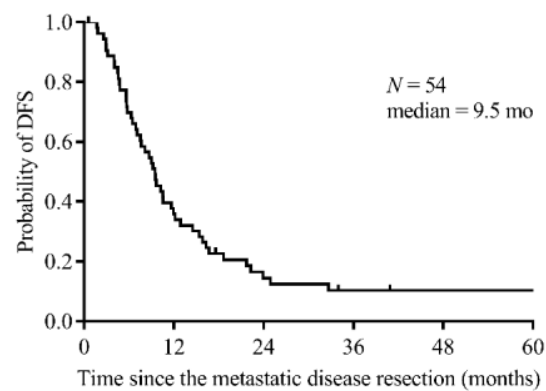

$1 D$

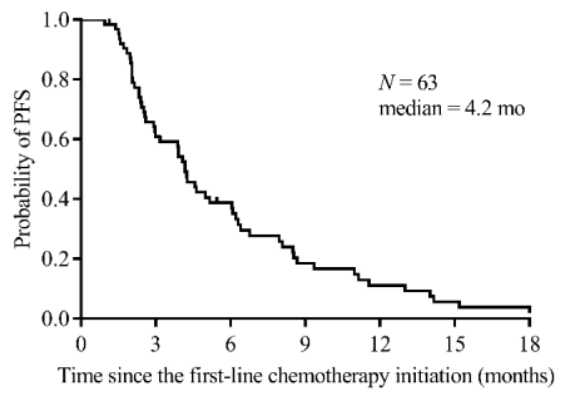

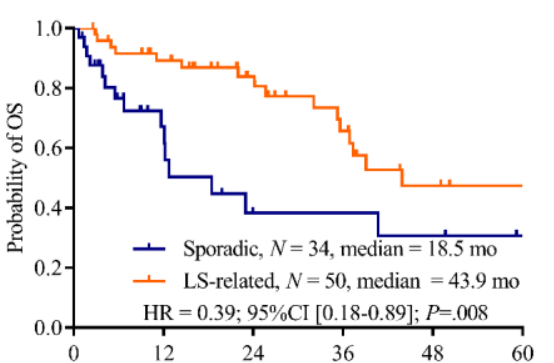

Time since the diagnostic of metastatic disease (months)
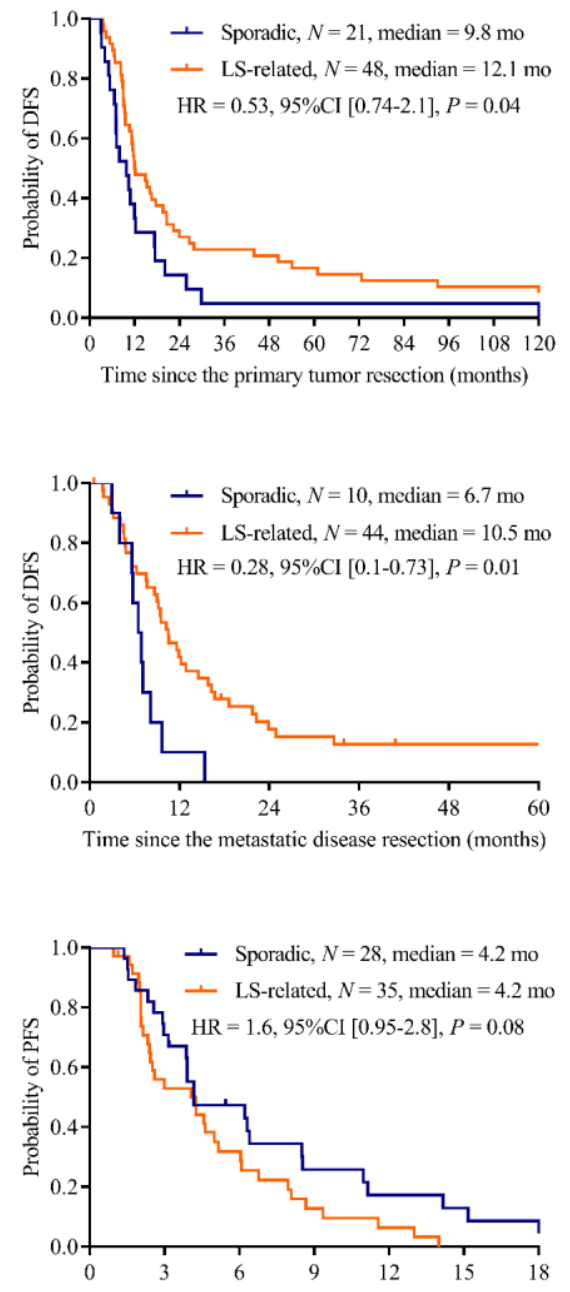

Time since the first-line chemotherapy initiation (months) 
Clinical and molecular characterization of hereditary and sporadic metastatic colorectal cancers harboring microsatellite instability / DNA mismatch repair deficiency

\section{Conflict of interest statement:}

$\mathrm{RC}$ has received travel expenses from Sanofi.

JBB declares honoraria from Amgen, Bayer, Celgene, Merck Serano and Sanofi, compensation from Amgen, Bayer, Merck Serono and Servier for consultancy and travel expenses from Merck Serano and Servier.

TA has received compensation from Bristol-Myers Squibb for consultancy and fees from Merck.

All remaining authors have declared no conflicts of interest. 
[1] Colle R, Cohen R, Cochereau D, Duval A, Lascols O, Lopez-Trabada D, et al. Immunotherapy and patients treated for cancer with microsatellite instability. Bull Cancer (Paris) 2017;104:42-51. doi:10.1016/j.bulcan.2016.11.006.

[2] Adar T, Rodgers LH, Shannon KM, Yoshida M, Ma T, Mattia A, et al. A tailored approach to BRAF and MLH1 methylation testing in a universal screening program for Lynch syndrome. Mod Pathol 2017.

[3] Newton K, Jorgensen NM, Wallace AJ, Buchanan DD, Lalloo F, McMahon RFT, et al. Tumour MLH1 promoter region methylation testing is an effective prescreen for Lynch Syndrome (HNPCC). J Med Genet 2014;51:789-96. doi:10.1136/jmedgenet-2014-102552.

[4] Parsons MT, Buchanan DD, Thompson B, Young JP, Spurdle AB. Correlation of tumour BRAF mutations and MLH1 methylation with germline mismatch repair (MMR) gene mutation status: a literature review assessing utility of tumour features for MMR variant classification. J Med Genet 2012;49:151-7. doi:10.1136/jmedgenet-2011-100714.

[5] Cunningham JM, Christensen ER, Tester DJ, Kim C-Y, Roche PC, Burgart LJ, et al. Hypermethylation of the hMLH1 promoter in colon cancer with microsatellite instability. Cancer Res 1998;58:3455-3460.

[6] Sargent DJ, Marsoni S, Monges G, Thibodeau SN, Labianca R, Hamilton SR, et al. Defective Mismatch Repair As a Predictive Marker for Lack of Efficacy of Fluorouracil-Based Adjuvant Therapy in Colon Cancer. J Clin Oncol 2010;28:3219-26. doi:10.1200/JCO.2009.27.1825.

[7] André T, Gramont A de, Vernerey D, Chibaudel B, Bonnetain F, Tijeras-Raballand A, et al. Adjuvant Fluorouracil, Leucovorin, and Oxaliplatin in Stage II to III Colon Cancer: Updated 10Year Survival and Outcomes According to BRAF Mutation and Mismatch Repair Status of the MOSAIC Study. J Clin Oncol 2015;33:4176-87. doi:10.1200/JCO.2015.63.4238.

[8] Tougeron D, Mouillet G, Trouilloud I, Lecomte T, Coriat R, Aparicio T, et al. Efficacy of Adjuvant Chemotherapy in Colon Cancer With Microsatellite Instability: A Large Multicenter AGEO Study. J Natl Cancer Inst 2016;108:djv438. doi:10.1093/jnci/djv438.

[9] Sinicrope FA, Foster NR, Thibodeau SN, Marsoni S, Monges G, Labianca R, et al. DNA Mismatch Repair Status and Colon Cancer Recurrence and Survival in Clinical Trials of 5-FluorouracilBased Adjuvant Therapy. J Natl Cancer Inst 2011;103:863-75. doi:10.1093/jnci/djr153.

[10] Brueckl WM, Moesch C, Brabletz T, Koebnick C, Riedel C, Jung A, et al. Relationship between microsatellite instability, response and survival in palliative patients with colorectal cancer undergoing first-line chemotherapy. Anticancer Res 2003;23:1773-7.

[11] Des Guetz G, Mariani P, Cucherousset J, Benamoun M, Lagorce C, Sastre X, et al. Microsatellite instability and sensitivitiy to FOLFOX treatment in metastatic colorectal cancer. Anticancer Res 2007;27:2715-2719.

[12] Tran B, Kopetz S, Tie J, Gibbs P, Jiang Z-Q, Lieu CH, et al. Impact of BRAF mutation and microsatellite instability on the pattern of metastatic spread and prognosis in metastatic colorectal cancer. Cancer 2011;117:4623-32. doi:10.1002/cncr.26086.

[13] Koopman M, Kortman GAM, Mekenkamp L, Ligtenberg MJL, Hoogerbrugge N, Antonini NF, et al. Deficient mismatch repair system in patients with sporadic advanced colorectal cancer. $\mathrm{Br} \mathbf{J}$ Cancer 2009;100:266-73. doi:10.1038/sj.bjc.6604867.

[14] Fujiyoshi K, Yamamoto G, Takenoya T, Takahashi A, Arai Y, Yamada M, et al. Metastatic Pattern of Stage IV Colorectal Cancer with High-Frequency Microsatellite Instability as a Prognostic Factor. Anticancer Res 2017;37:239-47.

[15] Venderbosch S, Nagtegaal ID, Maughan TS, Smith CG, Cheadle JP, Fisher D, et al. Mismatch Repair Status and BRAF Mutation Status in Metastatic Colorectal Cancer Patients: A Pooled Analysis of the CAIRO, CAIRO2, COIN, and FOCUS Studies. Clin Cancer Res 2014;20:532230. doi:10.1158/1078-0432.CCR-14-0332.

[16] Le DT, Uram JN, Wang H, Bartlett BR, Kemberling H, Eyring AD, et al. PD-1 Blockade in Tumors with Mismatch-Repair Deficiency. N Engl J Med 2015;372:2509-20. doi:10.1056/NEJMoa1500596.

[17] Overman MJ, McDermott R, Leach JL, Lonardi S, Lenz H-J, Morse MA, et al. Nivolumab in patients with metastatic DNA mismatch repair-deficient or microsatellite instability-high colorectal cancer (CheckMate 142): an open-label, multicentre, phase 2 study. Lancet Oncol 2017;0. doi:10.1016/S1470-2045(17)30422-9. 
[18] Giardiello FM, Allen JI, Axilbund JE, Boland CR, Burke CA, Burt RW, et al. Guidelines on Genetic Evaluation and Management of Lynch Syndrome: A Consensus Statement by the US Multi-Society Task Force on Colorectal Cancer. Gastroenterology 2014;147:502-26. doi:10.1053/j.gastro.2014.04.001.

[19] Stoffel EM, Mangu PB, Gruber SB, Hamilton SR, Kalady MF, Lau MWY, et al. Hereditary Colorectal Cancer Syndromes: American Society of Clinical Oncology Clinical Practice Guideline Endorsement of the Familial Risk-Colorectal Cancer: European Society for Medical Oncology Clinical Practice Guidelines. J Clin Oncol 2015;33:209-17. doi:10.1200/JCO.2014.58.1322.

[20] French AJ, Sargent DJ, Burgart LJ, Foster NR, Kabat BF, Goldberg R, et al. Prognostic Significance of Defective Mismatch Repair and BRAF V600E in Patients with Colon Cancer. Clin Cancer Res 2008;14:3408-15. doi:10.1158/1078-0432.CCR-07-1489.

[21] Taieb J, Zaanan A, Le Malicot K, Julié C, Blons H, Mineur L, et al. Prognostic Effect of BRAF and KRAS Mutations in Patients With Stage III Colon Cancer Treated With Leucovorin, Fluorouracil, and Oxaliplatin With or Without Cetuximab: A Post Hoc Analysis of the PETACC8 Trial. JAMA Oncol 2016;2:643. doi:10.1001/jamaoncol.2015.5225.

[22] Goldstein J, Tran B, Ensor J, Gibbs P, Wong HL, Wong SF, et al. Multicenter retrospective analysis of metastatic colorectal cancer (CRC) with high-level microsatellite instability (MSI-H). Ann Oncol 2014;25:1032-8. doi:10.1093/annonc/mdu100.

[23] Roth AD, Tejpar S, Delorenzi M, Yan P, Fiocca R, Klingbiel D, et al. Prognostic Role of KRAS and BRAF in Stage II and III Resected Colon Cancer: Results of the Translational Study on the PETACC-3, EORTC 40993, SAKK 60-00 Trial. J Clin Oncol 2010;28:466-74. doi:10.1200/JCO.2009.23.3452.

[24] Popovici V, Budinska E, Tejpar S, Weinrich S, Estrella H, Hodgson G, et al. Identification of a Poor-Prognosis BRAF-Mutant-Like Population of Patients With Colon Cancer. J Clin Oncol 2012;30:1288-95. doi:10.1200/JCO.2011.39.5814.

[25] Guinney J, Dienstmann R, Wang X, de Reyniès A, Schlicker A, Soneson C, et al. The consensus molecular subtypes of colorectal cancer. Nat Med 2015;21:1350-6. doi:10.1038/nm.3967.

[26] Balmaña J, Balaguer F, Cervantes A, Arnold D. Familial risk-colorectal cancer: ESMO Clinical Practice Guidelines. Ann Oncol 2013;24:vi73-vi80. doi:10.1093/annonc/mdt209.

[27] Le DT, Uram JN, Wang H, Bartlett BR, Kemberling H, Eyring AD, et al. Programmed death-1 blockade in mismatch repair deficient colorectal cancer. J Clin Oncol 2016;34:suppl; abstr 103.

\section{Supplementary methods}


Determination of MMR and microsatellite statuses

MMR and microsatellite statuses were systematically confirmed using immunohistochemistry and polymerase chain reaction in the laboratory team unit "microsatellite instability and cancer" from SaintAntoine Research Center (INSERM, UMR_S 938, 184 rue du Faubourg Saint-Antoine, Paris 75012, France).

Formalin-fixed, paraffin-embedded (FFPE) tumor specimen were stained with anti-MLH1, -PMS2, MSH2 and -MSH6 antibodies. Protein expression was considered negative when nuclear staining was absent in tumor cells. Non-tumor tissue was used as an internal control.

Tumor DNA was extracted from paraffin sections using the DNeasy Blood and Tissue DNA isolation kit (Qiagen, Hilden, Germany). MSI status was determined using Pentaplex PCR (BAT-25, BAT-26, NR-21, NR-24 and NR-27). All Pentaplex markers were analysed on an ABI PRISM 3100 Genetic Analyzer (Applied Biosystems, Foster City, California, USA). Microsatellite instability was defined by the instability of at least 2 markers.

\section{$\underline{M L H 1 \text { promoter methylation }}$}

Tumor DNA was treated with bisulfite (EZ DNA methylation-Gold kit, Zymo Research, Orange, CA). Amplicons were sequenced using Genetic Analyzer 3500XL and analyzed with Genemapper software (Applied Biosystems, Foster City, California, USA). Methylated (RKO) and unmethylated (ITS3) cell lines were used as control.

\section{$\underline{R A S \text { and } R A F \text { mutational status }}$}

KRAS (exons 2, 3 and 4), NRAS (exons 2 and 3) and BRAF (exon 15) mutational status was determined using real-time PCR (Light Cycler 480, Roche Diagnostic) and analyzed by pyrosequencing (Pyromark Q24, Qiagen). Discrepant cases were directly sequenced (ABI PRISM 3500L Dx Genetic Analyzer, Applied Biosystems, Foster City, California, USA).We did not analyzed samples for NRAS exon 4 mutations given their very low frequency in metastatic CRC [1]. 
Figure S1: Molecular definition of sporadic and inherited MMR deficiency

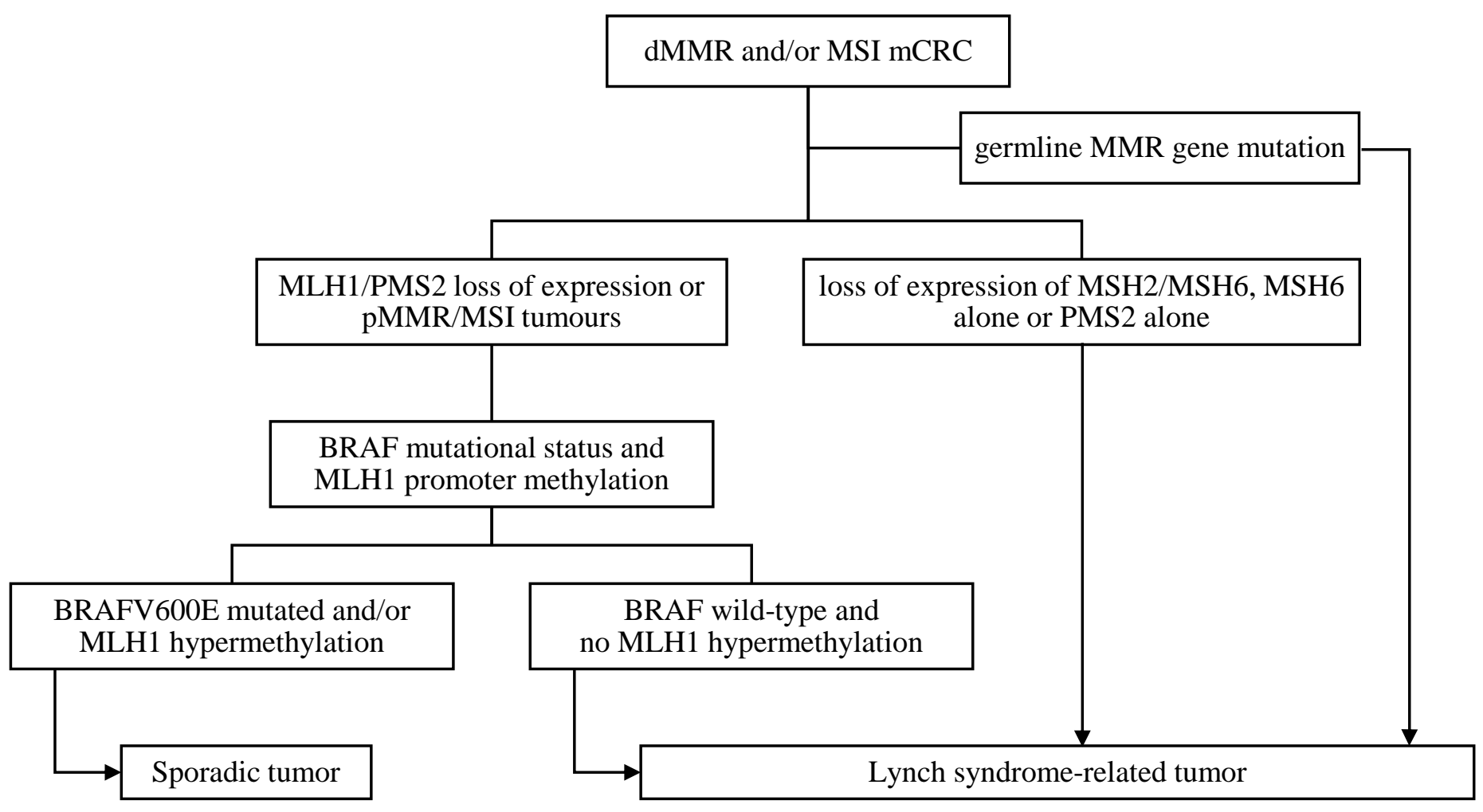




\section{$\underline{\text { Supplementary results }}$}

Table S1: Discrepancies between definitions of sporadic and inherited MMR deficiency in MLH1/PMS2-negative tumors

\begin{tabular}{|r|r|cc|c|}
\cline { 3 - 4 } \multicolumn{2}{c|}{} & \multicolumn{2}{c|}{ Molecular algorithm* } & \multirow{2}{*}{ Total } \\
\cline { 3 - 4 } \multicolumn{2}{c|}{} & Sporadic Lynch-like & \\
\hline Combined algorithm** & Sporadic & $42(66)$ & $9(14)$ & $51(80)$ \\
& Lynch-like & $5(8)$ & $8(13)$ & $13(20)$ \\
\hline & Total & $47(73)$ & $17(27)$ & $64(100)$ \\
\hline
\end{tabular}

Values in brackets correspond to percentages relative to patients with a loss of MLH1 and/or PMS2 expression in the tumor and no known MMR gene germline mutation $(N=$ 64)

* Tumors with BRAFV600E mutation and/or hypermethylated $M L H 1$ promoter were considered sporadic; $B R A F$ wild-type tumors with no hypermethylation of $M L H 1$ promoter were suspected to be related to Lynch syndrome

** All patients were considered to have sporadic dMMR CRC, except patients with a loss of MSH2/MSH6 expression, or a loss of MLH1 without BRAFV600E mutation and age inferior to 60 years, or if they met the Amsterdam II criteria in the absence of MMR immunochemistry [8].

Table S2: Correlation matrix (Spearman correlation coefficient)

\begin{tabular}{|c|c|c|c|}
\hline & $\begin{array}{c}\text { Origin of MMR } \\
\text { deficiency }\end{array}$ & $\begin{array}{c}\text { Age at metastatic } \\
\text { diagnosis }\end{array}$ & Resection of metastasis \\
\hline $\begin{array}{c}\text { Origin of MMR } \\
\text { deficiency }\end{array}$ & 1.00 & 0.581 & $\begin{array}{c}\mathrm{P}<0.0001 \\
\mathrm{~N}=84\end{array}$ \\
\hline $\begin{array}{c}\text { Age at metastatic } \\
\text { diagnosis }\end{array}$ & $\mathrm{P}<0.0001$ & 1.00 & $\mathrm{P}<0.0001$ \\
$\mathrm{~N}=84$
\end{tabular}

\section{Supplementary bibliography}

[1] Stintzing S, Miller-Phillips L, Modest DP, Fischer von Weikersthal L, Decker T, Kiani A, et al. Impact of BRAF and RAS mutations on first-line efficacy of FOLFIRI plus cetuximab versus FOLFIRI plus bevacizumab: analysis of the FIRE-3 (AIO KRK-0306) study. Eur J Cancer 2017;79:50-60. doi:10.1016/j.ejca.2017.03.023. 